Check for updates

Cite this: Chem. Commun., 2019, 55,4773

Received 28th February 2019,

Accepted 28th March 2019

DOI: $10.1039 / c 9 c c 01694 e$

rsc.li/chemcomm

\title{
Recognition and protection of glycosphingolipids by synthetic nanoparticle receptors $\dagger$
}

\author{
Roshan W. Gunasekara and Yan Zhao (D) *
}

\begin{abstract}
Nanoparticle receptors were synthesized through micellar imprinting to bind glycosphingolipids with 20-140 $\mu \mathrm{M}$ binding affinities, meanwhile distinguishing glycan composition, the number of acyl chains, and hydroxylation of acyl chains in the lipids. The strong binding enabled the receptors to protect their target glycolipids dispersed in lipid membranes from enzymatic degradation.
\end{abstract}

Glycosphingolipids are abundant in the plasma membrane of vertebrate tissues. In addition to promoting membrane packing and raft formation, they play important roles in cell signaling and regulation. ${ }^{1}$ Their metabolites, including sphingosine and lysosphingolipids, also have a plethora of biological functions including the regulation of cell growth, survival, immune cell trafficking, and development of inflammation and cancer. ${ }^{2-4}$ Although synthetic receptors for these molecules could have many potential applications, ${ }^{5-9}$ selective recognition of glycosphingolipids (and carbohydrates in general) has been difficult because of the strong solvation of glycans in water and the subtle difference of their structures, often by the stereochemistry of a single hydroxyl.

In this work, we report cross-linked micelles for the selective binding of several important glycosphingolipids (1-5). Among them, kerasin (1) and phrenosin (2) are major lipids in the brain. Their misregulation is related to Krabbe disease and their metabolite psychosine (3) is highly cytotoxic. ${ }^{10,11}$ Gaucher's disease causes abnormal accumulation of glucosylceramide 4 in the liver and spleen. ${ }^{12}$ Lactosylceramide (5) is a biological precursor to more complex glycolipids and is also involved in multiple signal transduction pathways. ${ }^{13}$ As natural lipids, the acyl groups of $1,2,4$, and 5 consist of mixtures of fatty acid chains with mostly 16-24 carbons and 0 or 1 degree of unsaturation. Psychosine has the acyl group removed.

Department of Chemistry, Iowa State University, Ames, Iowa 50011-3111, USA. E-mail: zhaoy@iastate.edu; Fax: +1-515-294-0105; Tel: +1-515-294-5845

$\dagger$ Electronic supplementary information (ESI) available: Experimental details, fluorescence titration curves, and additional figures. See DOI: 10.1039/ c9cc01694e
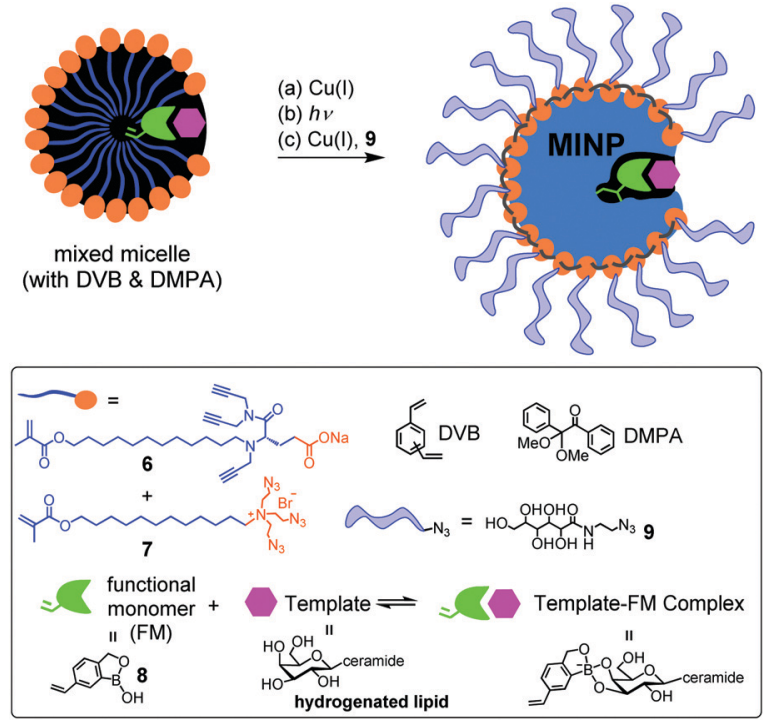

Scheme 1 Preparation of MINPs through micellar imprinting.

Scheme 1 shows the preparation of molecularly imprinted nanoparticle (MINP) receptors for these lipids. A glycosphingolipid, after hydrogenation (to prevent unsaturated lipid tails from participating in free radical polymerization), first forms a complex with functional monomer (FM) 8. The boroxole ${ }^{14,15}$ is known to form boronate esters with sugar in situ under micellar conditions ${ }^{16}$ similar to other boronic acid-derived sugarbinding molecules. ${ }^{17-19}$ The complex is incorporated into the mixed micelle of 6 and 7, along with DVB and DMPA (a photoinitiator). The micelle, with many alkynes and azides on the surface, is cross-linked on the surface by the $\mathrm{Cu}(\mathrm{I})$-catalyzed click reaction and then in the core by photo-induced free radical polymerization. In the final step, monoazide 9 is "clicked" onto the cross-linked micelle to enhance its hydrophilicity. ${ }^{20}$

The characterization of MINPs is reported in the ESI $\dagger$ (Fig. S2-S17). Their sizes $(4-5 \mathrm{~nm})$ were determined by DLS and had been confirmed by TEM. ${ }^{21,22}$ Their bindings with 1-5 were determined by isothermal titration calorimetry (ITC) and 
Table 1 Binding data for MINPs obtained by ITC

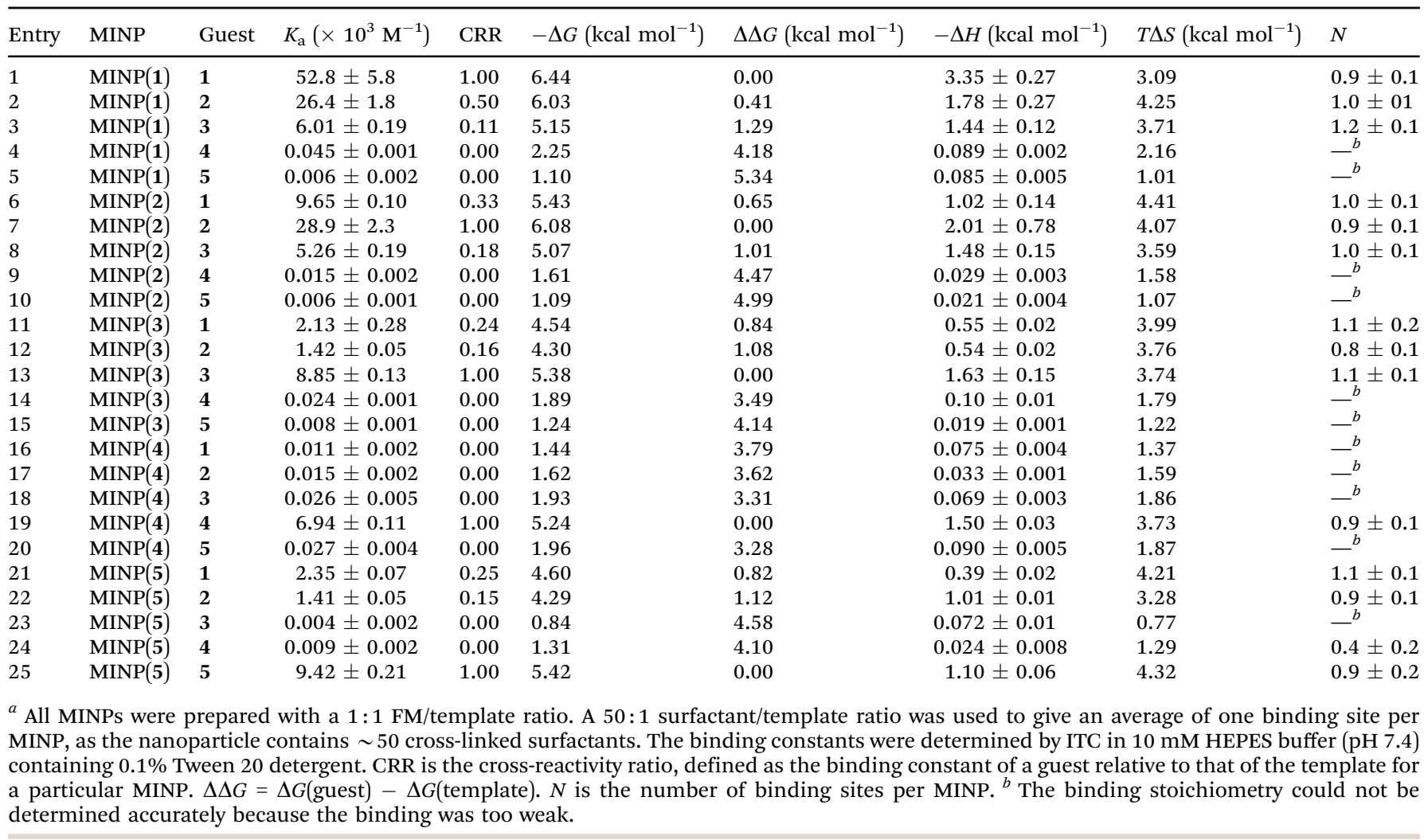

are summarized in Table 1 . Because the guest molecules were insoluble in water, we added $0.1 \%$ Tween 20 to the HEPES buffer in the ITC titrations-a common way to solubilize lipids. ${ }^{23}$

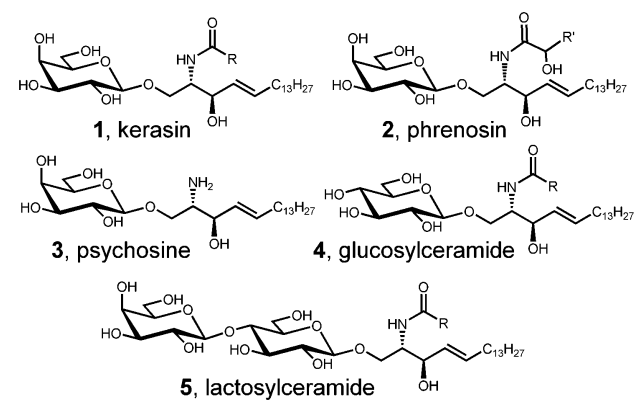

$\operatorname{MINP}(\mathbf{1})$, i.e., MINP prepared with kerasin 1 as the template, bound the template with $K_{\mathrm{a}}=52.8 \times 10^{3} \mathrm{M}^{-1}$ (entry 1). The binding constant is very competitive with those by natural lectin for a monosaccharide $\left(K_{\mathrm{a}}=10^{3}-10^{4} \mathrm{M}^{-1}\right){ }^{5,6}$ Consistent with successful imprinting, the CRR (cross-reactivity ratio) decreased significantly for other lipids. The very similar phrenosin 2 , having a single extra hydroxyl group on the acyl chain, gave a CRR of 0.50 (entry 2). Removal of the acyl chain was even less tolerated, affording a CRR of 0.11 for 3 (entry 3 ).

In aqueous solution, hydrophobic interactions dominate in the imprinting and binding of MINPs. ${ }^{24}$ When (hydrogenated) 1 was used as the template, everything including the hydrophobic tails should be imprinted. A change from 1 to 3, which misses a long acyl chain, however, only changed the binding energy of $\operatorname{MINP}(\mathbf{1})$ by $1.29 \mathrm{kcal} \mathrm{mol}^{-1}$ (entry 3). The small $\Delta \Delta G$ was inconsistent with the hydrophobic tail being involved in the binding. The conclusion is also supported by the overall binding energy of $\mathbf{1}$ by $\operatorname{MINP}(\mathbf{1})$. Because transfer of hexane to water costs $0.92 \mathrm{kcal} \mathrm{mol}^{-1}$ per methylene, ${ }^{25}$ burying two long hydrocarbon tails would have yielded a much larger binding energy than the observed value of $6.44 \mathrm{kcal} \mathrm{mol}^{-1}$. In our experiments, the hydrophobic tails of the lipid were solubilized by the hydrophobic core of the Tween micelle prior to binding. Apparently, once their hydrophobic needs were satisfied, there was no driving force to make the tails come out of the micelle and go into the imprinted sites (vide infra for additional discussion).

Gratifyingly, $\operatorname{MINP}(\mathbf{1})$ was extremely sensitive to the glycan, as 4 and 5 showed practically no binding. The selectivity in binding was $>1000$ for the glucosylceramide and lactosylceramide (entries 4 and 5). The selectivity was significant because 4 only differs from 1 in the stereochemistry of a single hydroxyl (on C4 of the glycoside). The large difference in binding suggests that this particular hydroxyl was involved in boronate formation. ${ }^{16,26}$ It is also significant that 5 was not bound at all. Even though the lactosylceramide contains a $\beta$-galactoside and thus has the correct diols to bind the boroxole, its large glycan precluded it from entering the small imprinted site created for $\mathbf{1}$.

MINP(2) displayed similar trends in binding and selectivity to $\operatorname{MINP}(1)$ (entries 6-10). For $\operatorname{MINP}(3)$, since its template does not have an acyl chain, it would not be able to bind $\mathbf{1}$ and $\mathbf{2}$ if it had to accommodate their long acyl group. Entries 11-12 show that $\operatorname{MINP(3)}$ exhibited a weaker but clearly observable binding 
for these acyl-containing lipids, thus supporting the earlier conclusion that the hydrophobic tails of the lipids stayed in the Tween micelle during binding and the binding was mainly derived from the glycan and its neighboring groups.

$\operatorname{MINP(4)}$ bound no other sphingolipids except its own template. The results once again highlighted the selectivity of MINPs for glycan, as $\mathbf{1}$ only differed from $\mathbf{4}$ in the stereochemistry of one hydroxyl.

Lactosylceramide 5 on its galactoside has the same boroxolebinding cis-3,4-diol ${ }^{16}$ as 1 . $\operatorname{MINP}(5)$, thus, has the appropriate boroxole in its binding pocket to bind the glycan of $\mathbf{1}$ even though the pocket was created for the disaccharide. Indeed, weaker but significant binding was obtained with this MINP for galactosylceramides 1 and 2 (entries 21 and 22). The fact that 1 and 2 showed appreciable binding suggests that boronate formation was key to the binding. It was initially puzzling to us that the galactosyl-containing psychosine $\mathbf{3}$ was bound extremely weakly (entry 23). However, given that the compound had both a shortened glycan and a missing acyl chain in comparison to $\mathbf{5}$, the difference might be just too large for $\operatorname{MINP}(5)$ to tolerate.

For all the bindings in Table 1, the entropic term $(T \Delta S)$ was positive (favorable). The result was reasonable because a large number of water molecules would be released to bulk upon binding. Interestingly, the binding enthalpy $(-\Delta H)$ was always the highest for the template over other guests, suggesting that correct boronate bond formation was important to the binding.

Tween is nonionic but MINP is anionic due to the excess of anionic surfactant 6 used in the preparation (Fig. S2, zetapotential $=-34.6 \mathrm{mV}$, ESI $\dagger$ ). Because many biological membranes are negatively charged, we could use their electric potential to modulate the binding of MINPs for a sphingolipid. This strategy could be very useful in biological applications, as alkynyl-functionalized cross-linked micelles can be functionalized with different surface groups to modulate their interactions with lipid membranes. ${ }^{27}$

Table 2 compares the binding of $\operatorname{MINP}(\mathbf{4})$ for 4 when solubilized by Tween and liposomes made from POPC (1-palmitoyl-2-oleoyl-sn-glycero-3-phosphocholine) and POPG (1-palmitoyl-2-oleoyl-sn-glycero-3-phosphoglycerol). POPC is a zwitterionic lipid and POPG is anionic. As shown by entries $2-5$, an increase of POPG in the membrane steadily decreased the binding, consistent with an electrostatic model.

Because both sphingolipids and their metabolites are important signaling and regulatory molecules, ${ }^{2-4}$ it is very useful to have chemical tools to control their enzymatic processing. Galactosyl lipids may be detected by Amplex Red assay, ${ }^{28}$ which uses a galactose oxidase to oxidize the sugar into the aldehyde derivative. The hydrogen peroxide byproduct generated meanwhile is used by horseradish peroxidase to oxidize Amplex Red into a fluorescent product, resorufin.

Fig. 1a shows four samples of Amplex Red assay after $35 \mathrm{~min}$ reaction time. As the photographs show, the enzymes produced more resorufin product (pink in color) with 1 solubilized in Tween 20 than in POPC. We attributed the difference to the different accessibility of the glycan in the micelle and in lipid membranes to galactose oxidase. Addition of $280 \mu \mathrm{M} \operatorname{MINP}(\mathbf{1})$ made the color much fainter, indicating slower oxidation of the galactoside. The last sample was a blank, showing no product formation in the absence of 1 . Fig. 1b shows the emission intensities of resorufin in the four samples. Based on the intensities, $280 \mu \mathrm{M} \operatorname{MINP}(\mathbf{1})$ slowed down the oxidation of the galactosylceramide by more than $50 \%$.

We also recorded the fluorescence spectra of a series of kerasin/Amplex Red samples with the concentration of $\operatorname{MINP}(\mathbf{1})$ varied from 0 to $280 \mu \mathrm{M}$ (Fig. S45a, ESI $\dagger$ ). As the concentration of the nanoparticle receptor increased, oxidation of $\mathbf{1}$ in the POPC lipid membranes decreased consistently. When we plotted the emission intensity at $585 \mathrm{~nm}$ at $35 \mathrm{~min}$ reaction time against the concentration of MINP, the intensities could be fitted nearly perfectly to a $1: 1$ binding isotherm (Fig. S45b, ESI $\dagger$ ). The apparent "binding constant" obtained was $K_{\mathrm{a}}=(5.42 \pm 0.75) \times 10^{3} \mathrm{M}^{-1}$. Even though this number was lower than the actual binding constant $\left(52.8 \times 10^{3} \mathrm{M}^{-1}\right)$ determined by ITC (Table 1 , entry 1$)$, the correlation does indicate that protection of the glycolipid from enzymatic degradation was a direct result of binding.

Natural lipids are often very expensive and sometimes difficult to obtain. Since our binding data suggested that MINPs

Table 2 Binding data for MINPs obtained by ITC under different solution conditions ${ }^{a}$

\begin{tabular}{|c|c|c|c|c|c|c|c|c|c|}
\hline Entry & MINP & Guest & Solution & $K_{\mathrm{a}}\left(\times 10^{3} \mathrm{M}^{-1}\right)$ & CRR & $-\Delta G\left(\mathrm{kcal} \mathrm{mol}^{-1}\right)$ & $-\Delta H\left(\mathrm{kcal} \mathrm{mol}^{-1}\right)$ & $T \Delta S\left(\mathrm{kcal} \mathrm{mol}^{-1}\right)$ & $N$ \\
\hline 1 & $\operatorname{MINP}(4)$ & 4 & $0.1 \%$ Tween-20 & $6.94 \pm 0.11$ & 1.00 & 5.24 & $1.50 \pm 0.03$ & 3.73 & $0.9 \pm 0.1$ \\
\hline 2 & $\operatorname{MINP}(4)$ & 4 & 1\% POPC & $5.98 \pm 0.72$ & 0.86 & 5.15 & $0.80 \pm 0.07$ & 4.35 & $1.1 \pm 0.1$ \\
\hline 3 & $\operatorname{MINP}(4)$ & 4 & 1\% $20: 1 \mathrm{POPC} / \mathrm{POPG}$ & $5.44 \pm 0.56$ & 0.78 & 5.09 & $1.31 \pm 0.06$ & 3.78 & $1.0 \pm 0.1$ \\
\hline 4 & $\operatorname{MINP}(4)$ & 4 & 1\% $5: 1 \mathrm{POPC} / \mathrm{POPG}$ & $1.77 \pm 0.06$ & 0.26 & 4.43 & $0.72 \pm 0.01$ & 3.71 & $1.0 \pm 0.1$ \\
\hline 5 & $\operatorname{MINP}(4)$ & 4 & $1 \%$ POPG & $0.045 \pm 0.008$ & 0.01 & 2.25 & $0.18 \pm 0.01$ & 2.07 & $-^{b}$ \\
\hline 6 & $\operatorname{MINP}(\mathbf{1 0})$ & 10 & $1 \% \mathrm{POPC}$ & $3.58 \pm 0.35$ & 1.00 & 4.84 & $1.39 \pm 0.15$ & 3.46 & $1.2 \pm 0.1$ \\
\hline 7 & $\operatorname{MINP}(\mathbf{1 0})$ & 4 & 1\% POPC & $1.71 \pm 0.08$ & 0.48 & 4.41 & $0.91 \pm 0.06$ & 3.49 & $1.0 \pm 0.1$ \\
\hline 8 & $\operatorname{MINP}(\mathbf{1 0})$ & 10 & HEPES buffer & $19.5 \pm 1.7$ & 5.45 & 5.85 & $1.60 \pm 0.11$ & 4.24 & $1.1 \pm 0.1$ \\
\hline 9 & $\operatorname{MINP}(11)$ & 11 & HEPES buffer & $30.3 \pm 1.1$ & 1.00 & 6.11 & $1.81 \pm 0.06$ & 4.30 & $0.9 \pm 0.1$ \\
\hline 10 & $\operatorname{MINP}(11)$ & 10 & HEPES buffer & $10.5 \pm 1.0$ & 0.35 & 5.48 & $4.30 \pm 0.21$ & 1.18 & $1.0 \pm 0.1$ \\
\hline 11 & $\operatorname{MINP}(11)$ & 10 & 1\% POPC & $2.01 \pm 0.06$ & 0.07 & 4.50 & $0.86 \pm 0.03$ & 3.64 & $1.0 \pm 0.1$ \\
\hline 12 & $\operatorname{MINP}(11)$ & 4 & 1\% POPC & $2.34 \pm 0.20$ & 0.08 & 4.59 & $0.61 \pm 0.05$ & 3.98 & $1.0 \pm 0.1$ \\
\hline
\end{tabular}

${ }^{a}$ All binding studies were determined by ITC in $10 \mathrm{mM}$ HEPES buffer (pH 7.4) containing $0.1 \%$ Tween-20 detergent. CRR is the cross-reactivity ratio, defined as the binding constant of a guest relative to that of the template for a particular MINP. $N$ is the number of binding sites per MINP.

${ }^{b}$ The binding stoichiometry could not be determined accurately because the binding was too weak. 
(a)

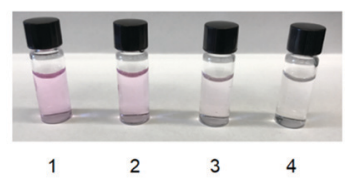

(b)

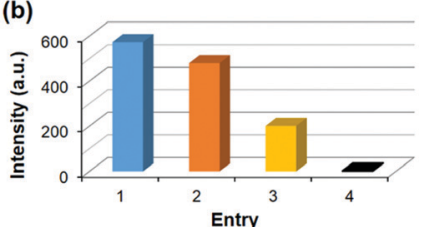

Fig. 1 (a) Photographs and (b) emission intensity at $585 \mathrm{~nm}$ of samples in the Amplex Red assay with (1) kerasin 1 in HEPES buffer with $0.1 \%$ Tween 20 , (2) 1 in HEPES buffer with 1\% POPC, (3) 1 in HEPES buffer with 1\% POPC and $280 \mu \mathrm{M} \mathrm{MIN} \mathrm{(6),} \mathrm{and} \mathrm{(4)} \mathrm{HEPES} \mathrm{buffer} \mathrm{1 \%} \mathrm{POPC} \mathrm{and} 280 \mu \mathrm{M}$ MINP (6).

recognized the sphingolipids mainly around their glycans, we decided to employ simple glycosides such as $\mathbf{1 0}$ and $\mathbf{1 1}$ to prepare MINPs for recognizing glucosylceramide 4.

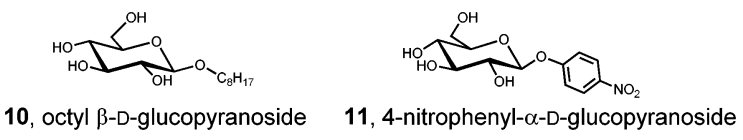

Table 2 shows that $\operatorname{MINP}(\mathbf{1 0})$ bound 4 indeed with a substantial binding constant $\left(K_{\mathrm{a}}=1710 \mathrm{M}^{-1}\right.$, entry 7$)$. Although the $K_{\mathrm{a}}$ value was half of that for the template itself (entry 6), and also weaker than that for 4 by $\operatorname{MINP}(4)$ under similar conditions $\left(K_{\mathrm{a}}=5980 \mathrm{M}^{-1}\right.$, entry 2$)$, the submillimolar affinity was impressive given that an analogue was used instead of the template. Because 10 was soluble in water, we also determined its binding by MINP (10) in HEPES buffer, without any additives (Tween or POPC). The binding constant was 5.45 times higher than that with POPC in the solution (entry 8 ). Thus, once the hydrophobic tail (i.e., octyl) was exposed to water prior to binding, it contributed strongly to the binding, unlike the situation when the glycolipids were solubilized by Tween or POPC.

Compound 11 has a different aglycon from 10. $\operatorname{MINP}(\mathbf{1 1})$, expectedly, bound its template strongly in buffer $\left(K_{\mathrm{a}}=30.3 \times\right.$ $10^{3} \mathrm{M}^{-1}$, entry 9). The imprinted pocket for the $p$-nitrophenyl group apparently was large enough for an octyl group to fold and fit in, ${ }^{29}$ as it bound 10 with $1 / 3$ of the binding constant (entry 10). Once the binding was measured in the presence of POPC, we lost the contribution from the hydrophobic aglycon again and obtained a lower $K_{\mathrm{a}}$ (entry 11). Importantly, glucosylceramide 4 was still bound with $K_{\mathrm{a}}>2000 \mathrm{M}^{-1}$ (entry 12). Thus, to prepare imprinted receptors for natural sphingolipids, we only need to focus on the key glycan instead of all the structure-a feature extremely useful in practical applications.

We thank NIGMS (R01GM113883) for financial support.

\section{Conflicts of interest}

There are no conflicts to declare.

\section{Notes and references}

1 C. Chalfant and M. Del Poeta, Sphingolipids as signaling and regulatory molecules, Springer Science, New York, N.Y., 2010.

2 Y. Hannun and R. Bell, Science, 1989, 243, 500-507.

3 M. Maceyka and S. Spiegel, Nature, 2014, 510, 58.

4 T. Wennekes, R. J. B. H. N. van den Berg, R. G. Boot, G. A. van der Marel, H. S. Overkleeft and J. M. F. G. Aerts, Angew. Chem., Int. Ed., 2009, 48, 8848-8869.

5 J. P. Kamerling and G.-J. Boons, Comprehensive glycoscience: from chemistry to systems biology, Elsevier, Amsterdam, Boston, 1st edn, 2007.

6 B. Wang and G.-J. Boons, Carbohydrate recognition: biological problems, methods, and applications, Wiley, Hoboken, N.J., 2011.

7 A. P. Davis and T. D. James, Carbohydrate Receptors, Wiley-VCH, Weinheim, 2005.

8 S. Jin, Y. Cheng, S. Reid, M. Li and B. Wang, Med. Res. Rev., 2010, 30, 171-257.

9 T. D. James, M. D. Phillips and S. Shinkai, Boronic acids in saccharide recognition, RSC Publishing, Cambridge, 2006.

10 L. Svennerholm, M. T. Vanier and J. E. Mansson, J. Lipid Res., 1980, 21, 53-64.

11 A. B. White, F. Galbiati, M. I. Givogri, A. Lopez Rosas, X. Qiu, R. van Breemen and E. R. Bongarzone, J. Neurosci. Res., 2011, 89, 352-364.

12 A. Dandana, S. Ben Khelifa, H. Chahed, A. Miled and S. Ferchichi, Pathobiology, 2016, 83, 13-23.

13 H. Nakamura, Y. Moriyama, T. Makiyama, S. Emori, H. Yamashita, R. Yamazaki and T. Murayama, J. Biol. Chem., 2013, 288, 23264-23272.

14 M. Dowlut and D. G. Hall, J. Am. Chem. Soc., 2006, 128, 4226-4227.

15 M. Bérubé, M. Dowlut and D. G. Hall, J. Org. Chem., 2008, 73, 6471-6479.

16 R. W. Gunasekara and Y. Zhao, J. Am. Chem. Soc., 2017, 139, 829-835.

17 G. Wulff and W. Vesper, J. Chromatogr., 1978, 167, 171-186.

18 X. Wu, Z. Li, X.-X. Chen, J. S. Fossey, T. D. James and Y.-B. Jiang, Chem. Soc. Rev., 2013, 42, 8032-8048.

19 S. D. Bull, M. G. Davidson, J. M. H. Van den Elsen, J. S. Fossey, A. T. A. Jenkins, Y. B. Jiang, Y. Kubo, F. Marken, K. Sakurai, J. Z. Zhao and T. D. James, Acc. Chem. Res., 2013, 46, 312-326.

20 Y. Zhao, Langmuir, 2016, 32, 5703-5713.

21 S. Fa and Y. Zhao, Chem. Mater., 2017, 29, 9284-9291.

22 S. Fa and Y. Zhao, Chem. - Eur. J., 2018, 24, 150-158.

23 S. Schuck, M. Honsho, K. Ekroos, A. Shevchenko and K. Simons, Proc. Natl. Acad. Sci. U. S. A., 2003, 100, 5795-5800.

24 J. K. Awino and Y. Zhao, J. Am. Chem. Soc., 2013, 135, 12552-12555.

25 M. H. Abraham, J. Am. Chem. Soc., 1982, 104, 2085-2094.

26 J. K. Awino, R. W. Gunasekara and Y. Zhao, J. Am. Chem. Soc., 2016, 138, 9759-9762.

27 X. Li and Y. Zhao, Bioconjugate Chem., 2012, 23, 1721-1725.

28 M. Fortelius and P. Mattjus, Chem. Phys. Lipids, 2006, 142, 103-110.

29 J. K. Awino and Y. Zhao, Org. Biomol. Chem., 2017, 15, 4851-4858. 